\title{
Comparison of Severe Complications after Acute Stanford Type B Aortic Dissection under Different Surgical Timing
}

\author{
Jie Wan, Jianhui Xu, Peng Li, Rui Li* \\ Department of Cardiology, Xiaogan Hospital, Wuhan University of Science and Technology, Xiaogan, China \\ Email: *xgyyxnk@163.com
}

How to cite this paper: Wan, J., Xu, J.H., $\mathrm{Li}, \mathrm{P}$. and Li, R. (2019) Comparison of Severe Complications after Acute Stanford Type B Aortic Dissection under Different Surgical Timing. International Journal of Clinical Medicine, 10, 128-134.

https://doi.org/10.4236/ijcm.2019.103013

Received: February 5, 2019

Accepted: March 5, 2019

Published: March 8, 2019

Copyright ( 2019 by author(s) and Scientific Research Publishing Inc. This work is licensed under the Creative Commons Attribution International License (CC BY 4.0).

http://creativecommons.org/licenses/by/4.0/

\begin{abstract}
Objective: To investigate the relationship between early intervention timing and complications of acute Stanford type B aortic dissection. Methods: The clinical data of 146 patients with acute stanford type B aortic dissection treated with transseptal stent for aortic endovascular repair (TEVAR) from January 2012 to October 2017 in Xiaogan Central Hospital were analyzed. The time was divided into 3 groups, including the onset to TEVAR time $\leq 48$ $\mathrm{h}$ group (41 cases in group A), the onset to TEVAR time $48 \mathrm{~h}-7 \mathrm{~d}$ group (56 cases in group B), the onset to TEVAR time $7 \mathrm{~d}-14 \mathrm{~d}$ group (49 cases in group C)). The clinical baseline data, the incidence of different complications during perioperative period, and the mortality rate at 30 days were compared between the three groups. Results: There were no significant differences in age, gender and comorbidities between the three groups (all $P>0.05$ ). Group A had a clearer indication of immediate intervention compared with group $\mathrm{B}$ and group $\mathrm{C}(P<0.05)$. The overall incidence of severe complications in group $\mathrm{C}$ was significantly lower than that in group $\mathrm{A}$ and group $\mathrm{B}$, and the difference was statistically significant $(P<0.05)$. There was no significant difference in reoperation rate and 30-day mortality between the 3 groups (all $P>$ 0.05). Conclusion: Early intervention of acute TBAD may increase the risk of serious complications after surgery, and the incidence of serious complications will gradually decrease over time; the reduction of severe complications after early grouping is not accompanied by Early mortality and reoperation rates were significantly reduced, and TEVAR treatment in some patients with dissection did not prevent dissection progression and rupture.
\end{abstract}

\section{Keywords}

Aortic Dissection, Thoracic Endovascular Aortic Repair, Surgical Timing, Serious Complications 


\section{Introduction}

Aortic dissection (AD) is one of the common high-risk chest pains in clinical work. In recent years, with the development of imaging techniques and clinical testing techniques, clinicians can quickly identify life-threatening causes of chest pain, especially with pulmonary embolism and the identification of acute coronary syndromes. The treatment of $\mathrm{AD}$ from Dake et al. [1] reported that the stent graft has been rapidly developed in the treatment of aortic dissection for several decades. Many recent mid-term follow-up results at home and abroad [2] [3] [4] also confirmed the success rate of TEVAR surgery. High, severe complication rate, and low trauma, endovascular isolation of the stanford type B aortic dissection (TBAD) has gradually replaced surgery as the first choice [5]. Although the evidence for the efficacy of T-type aortic dissection for TEVER treatment has accumulated, there is not much information available on the timing of early intervention for acute TBAD and the occurrence of postoperative serious complications. Foreign data [6] show that early intervention of acute TBAD may increase perioperative complications, and the occurrence of postoperative serious complications is related to the timing of intervention. There is currently no uniform understanding of how to perform early interventions to reduce early complications and reduce mortality in patients with acute complex TBAD or TBAD without clear complications. This study was a single-center retrospective study to investigate the relationship between the timing of early intervention in acute Stanford type B aortic dissection and postoperative serious complications.

\section{Methods}

\subsection{Patients}

Clinical data of 146 patients with acute TBAD who underwent aortic endovascular repair with a stent graft from January 2012 to October 2017 in Xiaogan Central Hospital. According to the time from onset to TEVAR, the patients were divided into the following three groups: the onset to TEVAR time $\leq 48$ group (41 cases in group A), the onset to TEVAR time $48 \mathrm{~h}-7 \mathrm{~d}$ group (group B 56), the onset to TEVAR time 7 - $14 \mathrm{~d}$ group (C Group of 49 cases). Inclusion criteria: 1) patients with acute TABD who were admitted to our hospital from January 2012 to October 2017; 2) treated with TEVAR during hospitalization; 3) complete aortic computed tomography angiography and related clinical data. Exclusion criteria: 1) history of surgical treatment of aortic disease; 2) type A aortic dissection and chronic type B aortic dissection; 3) intra-aortic hematoma (IMH); 4) Aortic atherosclerotic penetrating ulcer (PAU).

\subsection{Research Methods}

1) Systematic examination of medical records, radiographic data and clinical follow-up information of patients during hospitalization; 2) All patients with TBAD underwent TEVAR on the basis of standard medical therapy. The stan- 
dard of treatment success was that the proximal endometrial rupture was closed. No graft displacement and no residual endoleak, no significant branching vessels were closed and severe ischemic consequences; 3) Postoperative follow-up: Six months of follow-up for eligible patients, assessment of perioperative complications, reoperation rate, $30 \mathrm{~d}$ mortality, etc.

\subsection{Statistical Analysis}

Data analysis was performed using SPSS 19.0 statistical software. The measurement data were expressed as $\mathrm{x} \pm \mathrm{s}$, and the comparison between groups was analyzed by ANOVA. The comparison between the two groups was performed by LSD method; the count data was expressed as a percentage, and the comparison between groups was performed by chi-square test. $P<0.05$ was considered statistically significant.

\section{Results}

\subsection{Comparison of Baseline Data of Three Groups of Patients}

There were no significant differences in age, sex ratio and incidence of comorbidities between group A, group B and group C $(P>0.05)$; Group A had a clearer indication of immediate intervention compared with group $\mathrm{B}$ and group $\mathrm{C}(P$ $<0.05)$, See Table 1 for details.

\subsection{Comparison of Postoperative Complications and Recent Survival Rate}

There was no significant difference between the reoperation rate and the $30 \mathrm{~d}$

Table 1. Patient demographics and cause of TEVAR intervention.

\begin{tabular}{|c|c|c|c|c|c|c|c|}
\hline & \multicolumn{2}{|c|}{$\begin{array}{l}\text { Group A } \\
(\mathrm{N}=41)\end{array}$} & \multicolumn{2}{|c|}{$\begin{array}{l}\text { Group B } \\
(\mathrm{N}=56)\end{array}$} & \multicolumn{2}{|c|}{$\begin{array}{l}\text { Group C } \\
(\mathrm{N}=49)\end{array}$} & \multirow{2}{*}{$P$ value } \\
\hline & $\%$ & $\mathrm{n}$ & $\%$ & $\mathrm{n}$ & $\%$ & $\mathrm{n}$ & \\
\hline \multicolumn{8}{|l|}{ Patient demographics } \\
\hline Age $(\mathrm{y}, \bar{x} \pm s)$ & \multicolumn{2}{|c|}{$49.76 \pm 11.67$} & \multicolumn{2}{|c|}{$51.75 \pm 9.77$} & \multicolumn{2}{|c|}{$47.24 \pm 8.15$} & 0.068 \\
\hline Sex (male, \%) & 35 & 85.4 & 49 & 87.5 & 41 & 83.7 & 0.855 \\
\hline Hypertension & 36 & 87.8 & 40 & 71.4 & 36 & 73.5 & 0.136 \\
\hline Diabetes & 3 & 7.3 & 5 & 8.9 & 5 & 10.2 & 0.892 \\
\hline CHD & 2 & 4.9 & 4 & 7.1 & 5 & 10.2 & 0.628 \\
\hline \multicolumn{8}{|l|}{ Preoperative indication } \\
\hline Diameter $>45 \mathrm{~mm}$ & 16 & 39.0 & 13 & 23.2 & 10 & 20.4 & 0.104 \\
\hline Aura rupture & 6 & 14.6 & 3 & 5.4 & 2 & 4.1 & 0.123 \\
\hline Repeated pain & 7 & 17.1 & 8 & 14.3 & 4 & 8.2 & 0.428 \\
\hline Progression of dissection & 6 & 14.6 & 5 & 8.9 & 3 & 6.1 & 0.385 \\
\hline Insufficient perfusion of vital organs & 8 & 19.5 & 9 & 16.1 & 5 & 10.2 & 0.453 \\
\hline other & 7 & 17.1 & 21 & 37.5 & 26 & 53.1 & 0.002 \\
\hline
\end{tabular}


mortality rate in group A compared with group B and group $\mathrm{C}(P>0.05)$. The overall incidence of severe complications in group $C$ was significantly lower than that in group A and group B, and the difference was statistically significant $(P<$ 0.05). See Table 2 for details.

\section{Discussion}

Aortic dissection (AD) often leads to immediate life threats due to rapid disease progression. It is highly valued in clinical diagnosis and treatment. The untreated acute $\mathrm{AD} 24 \mathrm{~h}$ mortality rate is about $33 \%$, and the mortality rate within 48 hours is as high as 50\% [7] [8]. Usually, the age of onset of $\mathrm{AD}$ is $48-67$ years old, and the ratio of male to female is $(2-5): 1$ [9]. Hypertension is the most common cause of $\mathrm{AD}[10]$. This study also showed that there were significant gender differences in 146 patients with stanford type B aortic dissection. The overall male to female ratio was approximately $6: 1$. More than $70 \%$ of patients had hypertension, and 40 to 65 years of age were the prevalence of TBAD. segment.

TEVAR has been widely accepted for the treatment of Stanford B-type dissection. Compared with surgical open surgery and standard medical treatment, its comprehensive advantages have become increasingly prominent. In 2014, the European Society of Cardiology's guidelines for the diagnosis and treatment of aortic diseases used TEVAR as the first choice for complex TBAD. Treatment (I, C), non-complex TBAD may also consider TEVAR treatment (IIa, B). However, the INSTEAD trial [11] and the ADSORB study [12] demonstrated that patients with acute noncomplexity TBAD had better aortic remodeling with TEVAR than with drug therapy alone, and patients who underwent early TEVAR intervention in the INSTEAD trial were 5 years later. The rate of aortic-related mortality and dissection was much lower than that of the drug-only group. Complex TBAD should be considered when aortic aura rupture (blood thoracic, mediastinal hematoma, etc.), major organ hypoperfusion, recurrent pain, refractory

Table 2. Major complications.

\begin{tabular}{cccccccc}
\hline & Group A $(\mathrm{N}=41)$ & Group B $(\mathrm{N}=56)$ & Group C (N = 49) & $P$ value \\
\hline Major complications & $\%$ & $\mathrm{n}$ & $\%$ & $\mathrm{n}$ & $\%$ & $\mathrm{n}$ & \\
New kidney failure & 12.2 & 5 & 7.1 & 4 & 2.0 & 1 & 0.164 \\
Retrograde sandwich & 4.9 & 2 & 0.0 & 0 & 0.0 & 0 & 0.075 \\
Paraplegia & 4.9 & 2 & 1.8 & 1 & 2.0 & 1 & 0.611 \\
Brain infarction & 2.4 & 1 & 1.8 & 1 & 0.0 & 0 & 0.577 \\
Lower limb ischemia & 4.9 & 2 & 1.8 & 1 & 0.0 & 0 & 0.263 \\
Gastrointestinal ischemia & 7.3 & 3 & 3.6 & 2 & 0.0 & 0 & 0.164 \\
total & 36.6 & 15 & 16.1 & 9 & 4.1 & 2 & 0.0002 \\
Reoperation rate & 12.2 & 5 & 7.1 & 4 & 2.0 & 1 & 0.164 \\
30 d mortality & 14.6 & 6 & 5.4 & 3 & 4.1 & 2 & 0.123 \\
\hline
\end{tabular}


hypertension, early dissection of the dissection, and persistent progression of the lesion. In this study, the proportion of acute complex TBAD in group A was significantly higher than that in group $\mathrm{B}$ and $\mathrm{C}(P<0.05)$, indicating that the clinical situation of patients with early TEVAR intervention was more complicated, but it was caused by complex clinical conditions and varied. There is no very effective risk stratification tool and assessment tool for aortic anatomy. Studies [13] have shown that when TBAD patients have the above clinical manifestations, the mortality rate is significantly increased. Therefore, early intervention of TEVAR on the basis of standard drug therapy is particularly important for such patient populations.

Regarding severe postoperative complications, the overall incidence of severe complications in group $\mathrm{C}$ was significantly lower than that in group $\mathrm{A}$ and group $\mathrm{B}(P<0.05)$. The overall incidence of severe complications in group $\mathrm{B}$ was lower than that in group. Group A $(P<0.05)$, group $\mathrm{C}$ was lower than group $\mathrm{B}(P<$ $0.05)$, indicating that the overall postoperative serious complication rate of patients with acute TBAD undergoing TEVAR treatment gradually decreased over time. The probable cause is that the proportion of acute complex TBAD in the earliest stage of intervention is higher, followed by acute vascular inflammation and edema, aortic intima fragile and easy to tear, and TEVAR is more prone to endoleak, new breach formation, and dissection. Postoperative complications such as retrograde tearing and aortic rupture [14]. However, we found that with the reduction of the incidence of serious complications, the reoperation rate and early mortality of the corresponding patients did not decrease significantly $(P>$ $0.05)$, indicating that patients with acute TABD are a diverse group, simple staging and Early intervention problems in some patients with acute TABD who are facing immediate life threats cannot be resolved. Complex clinical conditions and variable aortic anatomy suggest the need for individualized treatment.

At the follow-up, we found that the risk factors for postoperative complications of aortic dissection were mainly age, underlying disease, and coverage of the left subclavian artery. Postoperative ischemic stroke is closely related to preoperative and postoperative hemodynamic changes; endoleak is the result of the combined effects of dissection lesions, vascular conditions, and artificial vascular stent grafts. Two cases of postoperative ischemic stroke were caused by atherosclerotic plaques in the internal carotid artery and subclavian artery, plus surgical procedures and hemodynamic changes. These cases provide a reference for our future work. Postoperative type II and III endoleaks generally require close follow-up without special treatment. For patients with TBAD with stable disease, TEVAR should be treated as soon as possible after 1 week (Figure 1). Special attention should be paid to the preoperative renal function of the patient, because the application of intraoperative contrast agent will further aggravate renal insufficiency or failure. Detailed evaluation and formulation of the plan before surgery, intraoperative standard operation, good stent selection, and strict follow-up after surgery are beneficial to reduce or reduce the occurrence of postoperative complications. 


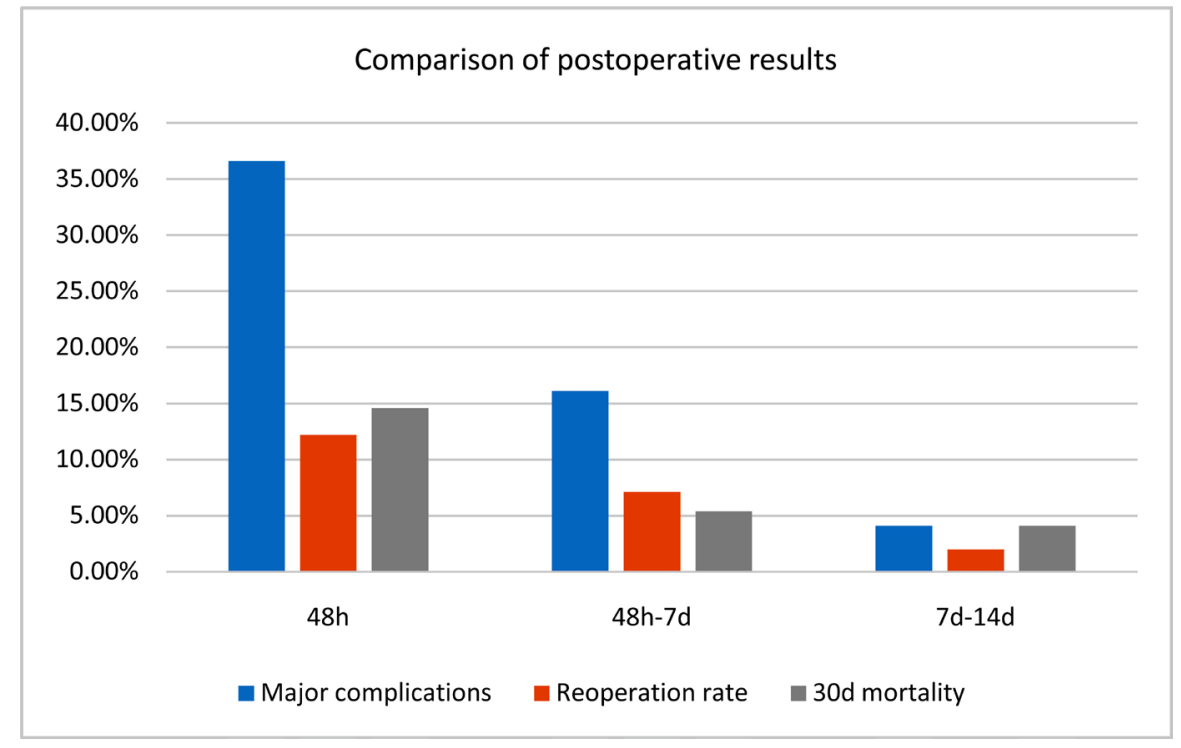

Figure 1. Short-term prognosis.

\section{Conclusion}

In summary, early intervention of acute TBAD will increase the risk of serious complications after surgery, and the incidence of serious complications will gradually decrease over time. The reduction in postoperative severe complications in the early grouping was not associated with a significant reduction in early mortality and reoperation rates. TEVAR treatment in some patients with dissection did not prevent dissection progression and rupture. At present, it is difficult to classify and effectively classify patients with acute TBAD. Early intervention also faces the complex clinical environment and the test of a variety of aortic anatomy. Early intervention strategies for patients with acute TBAD have yet to be refined in a multicenter, prospective, randomized controlled trial.

\section{Conflicts of Interest}

The authors declare no conflicts of interest regarding the publication of this paper.

\section{References}

[1] Dake, M.D., Kato, N., Mitchell, R.S., et al. (1999) Endovascular Stent-Graft Placement for the Treatment of Acute Aortic Dissection. The New England Journal of Medicine, 340, 1546-1552. https://doi.org/10.1056/NEJM199905203402004

[2] Pang, D., Hildebrand, D. and Bachoo, P. (2015) Thoracic Endovascular Repair (TEVAR) versus Open Surgery for Blunt Traumatic Thoracic Aortic Injury. The Cochrane Library, John Wiley \& Sons, CD006642.

[3] Lee, H.C., Joo, H.C., Lee, S.H., et al. (2015) Endovascular Repair versus Open Repair for Isolated Descending Thoracic Aorticaneursm. Yonsei Medical Journal, 56, 904912. https://doi.org/10.3349/ymj.2015.56.4.904

[4] Li, D.L., Zhang, H.K., Chen, X.D., et al. (2016) Thoracic Endovascular Aortic Repair for Type B Aortic Dissection: Analysis among Acute, Subacute and Chronic Pa- 
tients. Journal of the American College of Cardiology, 67, 1255-1257.

https://doi.org/10.1016/j.jacc.2015.12.044

[5] Writing Committee, Riambau, V., Bockler, D., Brunkwall, J., et al. (2017) Editor's Choice e Management of Descending Thoracic Aorta Diseases: Clinical Practice Guidelines of the European Society for Vascular Surgery (ESVS). European Journal of Vascular and Endovascular Surgery, 53, 4-52. https://doi.org/10.1016/j.ejvs.2016.06.005

[6] Miyairi, T., Miyata, H., Chiba, K., et al. for the Japan Adult Cardiovascular Database Organization (2018) Influence of Timing after Thoracic Endovascular Aortic Repair for Acute Type B Aortic Dissection. The Annals of Thoracic Surgery. https://doi.org/10.1016/j.athoracsur.2017.11.054

[7] Moon, M.R. (2009) Approach to the Treatment of Aortic Dissection. Surgical Clinics of North America, 89, 869-893. https://doi.org/10.1016/j.suc.2009.05.003

[8] Ohlmann, P., Faure, A., Morel, O., et al. (2006) Diagnostic and Prognostic Value of Circulating D-Dimers in Patients with Acute Aortic Dissection. Critical Care Medicine, 34, 1358-1364. https://doi.org/10.1097/01.CCM.0000216686.72457.EC

[9] Lo, R.C., Bensley, R.P., Hamdan, A.D., et al. (2013) Gender Difference in Abdominal Aortic Aneurysm Presentation, Repair, and Mortality in the Vascular Study Group of NewEngland. Journal of Vascular Surgery, 57, 1261-1268. https://doi.org/10.1016/j.jvs.2012.11.039

[10] Fukuda, S., Watanabe, H., Iwakura, K., et al. (2015) Multicenter Investigations of the Prevalene of Abdominal Aortic Aneurysm in Elderly Japanese Patents with Hypertension. Circulation Journal, 79, 524-529.

[11] Nienaber, C.A., Rousseau, H., Eggebrecht, H., et al. (2009) Randomized Comparison of Strategies for Type B Aortic Dissection: The Investigation of Stent Grafts in Aortic Dissection (INSTEAD) Trial. Circulation, 120, 2519-2528. https://doi.org/10.1161/CIRCULATIONAHA.109.886408

[12] Brunkwall, J., Kasprzak, P., Verhoeven, E., et al. (2014) Endovascular Repair of Acute Uncomplicated Aortic Type B Dissection Promotes Aortic Remodelling: 1 Year Results of the ADSORB Trial. European Journal of Vascular and Endovascular Surgery, 48, 285-291. https://doi.org/10.1016/j.ejvs.2014.05.012

[13] Tolenaar, J.L., Froehlich, W., Jonker, F.H., et al. (2014) Predicting In-Hospital Mortality in Acute Type B Aortic Dissection: Evidence from International Registry of Acute Aortic Dissection. Circulation, 130, 45-50. https://doi.org/10.1161/CIRCULATIONAHA.113.007117

[14] Mehta, M., Paty, P. and Bhaghtwar, P. (2015) False Lumen Embolization during TEVAR for Complicated Aortic Dissections Is Associated with a Lower 30-Day Mortality and Improved Longterm Survival. Journal of Vascular Surgery, 62, 817. https://doi.org/10.1016/j.jvs.2015.06.128 